\title{
THE SUPERMEMBRANE WITH CENTRAL CHARGES: $(2+1)$-D NCSYM, CONFINEMENT AND PHASE TRANSITION
}

\author{
L BOULTON $^{1}$, M P GARCIA DEL MORAL ${ }^{2}$ \\ AND A RESTUCCIA ${ }^{3}$
}

\begin{abstract}
The spectrum of the bosonic sector of the $D=11$ supermembrane with central charges is shown to be discrete and with finite multiplicities, hence containing a mass gap. The result extends to the exact theory our previous proof of the similar property for the $S U(N)$ regularised model and strongly suggest discreteness of the spectrum for the complete Hamiltonian of the supermembrane with central charges. This theory is a quantum equivalent to a symplectic non-commutative super-Yang-Mills in $2+1$ dimensions, where the space-like sector is a Riemann surface of positive genus. In this context, it is argued how the theory in $4 D$ exhibits confinement in the $N=1$ supermembrane with central charges phase and how the theory enters in the quark-gluon plasma phase through the spontaneous breaking of the centre. This phase is interpreted in terms of the compactified supermembrane without central charges.
\end{abstract}

Date: 15 th July 2007. 


\section{INTRODUCTION}

1.1. Motivation. An important step towards a better understanding of the non-perturbative approach to superstring theory is the nonperturbative treatment of $D=11$ supermembranes [1]. The quantisation of the latter, when it is embedded on Minkowski space-time, was studied in 2, 3, 4, 5] in terms of a quantum mechanical maximally supersymmetric $S U(N)$ Yang-Mills matrix models. This was also considered in a different context in [6]. In the seminal work [2], it was shown that the spectrum of the $S U(N)$ regularised supersymmetric Hamiltonian is continuous, consisting of the interval $[0, \infty)$. Remarkably, the spectrum of the corresponding bosonic Hamiltonian, equivalent to the dimensional reduction of $D=10$ super-Yang-Mills to $0+1$ space-time, is discrete [7, 8]. However its configuration space comprises singular string-like spikes, which, along with supersymmetry, render the spectrum continuous.

The validity of the $S U(N)$ regularisation is justified by the fact that the structure constants of the area-preserving diffeomorphisms, the gauge symmetry of the supermembrane in the light cone gauge, are equal to the large $N$ limit of the $S U(N)$ structure constants. The characterisation of the spectrum was performed in the $S U(N)$ regularised model, but we are not aware of any result concerning the large $N$ limit of the spectrum.

The supermembrane was interpreted as a many-body object. In this interpretation, the string-like spikes may connect different membranes without changing the energy of the system, in distinction to the standard situation in string theory. For a review see [10]. The supermembrane embedded on a target space with a compact sector was analysed in [9]. Although an $S U(N)$ regularisation was not obtained, it was argued that the same qualitative features of the spectrum remain valid.

The $D=11$ supermembrane with non-trivial central charges was introduced in [11. The configuration space of this model is restricted by a topological condition. This restriction implies the existence of a non-trivial central charge on the SUSY algebra of the supermembrane. From a geometrical point of view, the topological condition determines a non-trivial $U(1)$ principal bundle over the worldvolume whose canonical connections, $U(1)$ monopoles, define minimal immersions into the compactified sector of the target space. These immersions describe the wrapping of the supermembrane on a calibrated submanifold of the target space. An interesting case is $M_{4} \times T^{d} \times\left(S^{1}\right)^{7-2 g}$, where $M_{4}$ represents a Minkowski space-time in the light-cone gauge and $g=1,2,3$ 
is the genus of the 2-torus. See [16] for the case $M_{7} \times T^{4}$. The resulting theory preserves an $N=1$ supersymmetry in those dimensions.

The supermembrane with non-trivial central charges does not contain string-like spikes and it admits an $S U(N)$ regularisation [12]. The bosonic potential of this model increases towards infinity as we move away from zero in the configuration space, ensuring a compact resolvent for the bosonic Hamiltonian [13]. The spectrum of the regularised Hamiltonian is discrete, with finite multiplicity [14] and its heat kernel can be defined rigorously by a process described in [15].

For a $g \geq 2$ base manifold, this supermembrane admits an interpretation in terms of an orthogonal intersection of a suitable number of genus 1 supermembranes with non-trivial central charge [16]. In the type $I I A$ picture, the theory compactified on a $T^{2}$, i.e. $g=1$, may be viewed as a bundle of $D 2-D 0$ bound state theories where $D 0$ monopole charges are induced by non-constant fluxes on the D2 [17]. Extensions to $S U(N)$ interacting supermembranes may be considered as in [18].

In the cases where a minimal immersion from the base manifold into the compact sector of the target space can be established (the former is a Riemann surface of genus $g$ ), the topological restriction can be solved, and the supermembrane with non-trivial central charges is equivalent (as a quantum field theory) to a symplectic non-commutative superYang-Mills theory (NCSYM) [19, 20]. This is the case, for instance, when the base manifold is a genus $g$ Riemann surface and the compact sector of the target space is a flat $T^{2 g}$ torus. The symplectic structure is determined by the minimal immersion, and describes the curvature of $U(1)$ monopole connections.

The BFSS conjecture takes the D0 action as a fundamental action 31. This is precisely the same Hamiltonian than the $\mathrm{SU}(\mathrm{N})$ regularised Hamiltonian of the $D=11$ supermembrane in the light cone gauge [2]. Its extension to interesting compactified target spaces as the BMN model 32 includes Chern-Simons and mass terms, preserving the PP wave supersymmetry, allowing the existence of stable vacum solutions. In the same sense, the topological condition on the $D=11$ supermembrane with central charges give rise to mass terms which render the theory stable. However, the topological properties of the base manifold plays a fundamental role in the large $N$ limit analysis of the regularised supermembrane. This geometrical structure is missing in the matrix model, where no rigorous analysis of the large $N$ limit has been obtained.

Non-commutative Yang-Mills theories have been considered as toy models for gravity [21] and [22]. The relation between supergravity 
and non-commutative Yang-Mills become natural in the context of supermembranes, since they are embedded on a target space which must be a solution of $D=11$ supergravity. Moreover the supermultiplet of $D=11$ supergravity has been conjectured to be the ground state of the theory.

In the context of string theory, NCSYM appears in a very natural manner by wrapping D-branes [23]. A super-Yang-Mills theory on a non-commutative torus is naturally related to the compactification of a matrix theory on a dual torus with a constant $C_{3}$ field, see for example, [24, 25, 26, 27]. The non-commutative Yang-Mills theory in a flat space with a rational non-commutative parameter is related to ordinary Yang-Mills theories with magnetic flux through Morita equivalence, [28]. By comparing ordinary Yang-Mills theories and non-commutative ones, it was found in 29] that both theories share the same degrees of freedom in the IR limit. However these degrees are redistributed differently in both theories in the UV limit.

It was argued in [27, 30] that it should be the non-commutative theory the better suited for describing the IR limit and the commutative theory the one that best recovers the UV limit.

Yang-Mills theories with boundary modelling QCD where extensively studied under the common name of "bag models" [41]. super-YangMills theories behave differently at extremal energies since they are either in the confined or in the screening phase. For the bosonic theory, the confined phase occurs at low temperatures where vector-like gluons form singlet bound states of colour called glueballs, see [33].

The low temperature regime is characterised by the dynamics of gluons. At high temperatures the gluons do no give rise to bound states but rather to a plasma that constitute the screening phase. If the fermions are introduced in the theory, at low energies they feel a binding force and at high energy they remain free, 35. These two extremal regimes are thought in general to be separated by a phase transition occurring when a global symmetry of the theory breaks. This phase transition is related to the spontaneous breaking of the centre, [35, 36]. Furthermore, according to [35, 36], this symmetry is conjectured to be of a topological nature due to magnetic monopoles or instantons.

The role of the centre has been considered to a large extent, cf. e.g. [33, 37, 38]. According to the results of [39], the instanton gas picture is only appropriate in those cases where the topological charge is discrete. Otherwise the correct picture would be the monopole. It is this latter case, where the confinement should appear, [39], as it is for $D=4$ and $N=1$ in the supermembrane with central charges. 
Connections between membrane theory and Yang-Mills theories have been considered in a different context in [40]. In [42] the critical behaviour of a gauge theory in the de-confined phase is related with the behaviour of a scalar which has a symmetry induced by the centre of the group. The phase transition occurs when the topological defect is metastable and decays as a consequence of quantum processes.

1.2. Aims and scopes of the present paper. Our main goal is to demonstrate that the bosonic Hamiltonian of the supermembrane with non-trivial central charges has a discrete spectrum with finite multiplicity. Based on this observation we will show strong evidence confirming that the spectrum of the supersymmetric Hamiltonian possesses the same qualitative properties. Consequently, the $N=1$ NCSYM theory in $2+1 \mathrm{D}$ is equivalent to the supermembrane with non-trivial central charge has the same spectral properties.

We also prove that, in the semiclassical regime, the large $N$ limit of the eigenvalues of the regularised Hamiltonian converge to those of the supermembrane with central charge. The spectrum of the Hamiltonian exhibits a mass gap. The scalar fields acquire a mass induced by the central charge of the SUSY algebra. One may also argue that this mass is induced by the centre $Z(2)$ of the symplectic group associated to the central charge. Supermembranes with central charge and $N=1$ in $4 \mathrm{D}$ may then be interpreted as the IR phase of a more general theory. This is achieved by increasing the energy the centre breaks spontaneously, so that the transition phase occurs in a screening phase. This should correspond to an $N=4$ compactified supermembrane without central charges.

In Section 2, we describe the Hamiltonian of the supermembrane with central charges and its semiclassical regime. In Section 3 we establish the main results of the present paper, a crucial operator bounds connecting the exact bosonic Hamiltonian and the semiclassical Hamiltonian. This operator bound, see (22), allows us to deduce spectral properties of the exact bosonic Hamiltonian from those of the semiclassical one discussed in Section 2. Natural questions arises concerning the large $N$ limit of the regularised Hamiltonian. We address these questions in Section 4. In the final Section 5 we consider confinement properties of the theory in terms of the centre of the group both at the exact and the regularised level. We also discuss the transition phase to de-confinement and give an interpretation in terms of the supermembrane. An appendix is included, where we explicitly compute the $S U(N)$ gauge symmetry in the regularisation model. 


\section{The SUPERMEMBRAnes With CENTRAL CHARGES}

In this section we analyse the exact action of the supermembrane with central charges as well as its semiclassical approximation.

Let the $D=11$ supermembrane be defined in terms of a base manifold, a $g=1$ Riemann surface $\Sigma$, and a target space $M_{9} \times S^{1} \times S^{1}$. Consider its formulation in the light cone gauge where the directions $X^{+}, X^{-}, P_{+}$and $P_{-}$have been removed in the standard manner [4]. The canonically reduced Hamiltonian has the expression

$$
\int_{\Sigma} \sqrt{W}\left(\frac{1}{2}\left(\frac{P_{M}}{\sqrt{W}}\right)^{2}+\frac{1}{4}\left\{X^{M}, X^{N}\right\}^{2}+(\text { fermionic terms })\right)
$$

subject to the condition

$$
\oint_{\mathcal{C}} \frac{P_{M}}{\sqrt{W}} \mathrm{~d} X^{M}=0
$$

Here and below $M, N=1, \ldots, 9$. The integral in (11) is the generator of an area preserving diffeomorphism of $\Sigma$ for $\mathcal{C}$ any given closed path. This constraint may be expressed as a local condition

$$
\left\{P_{M}, X^{M}\right\} \equiv \epsilon^{a b} \partial_{a}\left(\frac{P_{M}}{\sqrt{W}}\right) \partial_{b} X^{M}=0,
$$

which generates area preserving diffeomorphisms associated to exact one-forms coupled to the global constraint

$$
\oint_{C_{i}} \frac{P_{M}}{\sqrt{W}} \mathrm{~d} X^{M}=0, \quad i=1,2,
$$

where $C_{1}$ and $C_{2}$ form of a basis of homology on $\Sigma$ which generates area preserving diffeomorphisms associated to harmonic one-forms.

The scalar density $\sqrt{W}$ is present in expression (2) as a consequence of the gauge fixing procedure and it is preserved by the diffeomorphisms mentioned above. Let us now impose some topological restrictions on the configuration space which completely characterise the $D=11$ supermembrane with non-trivial central charge generated by the wrapping on the compact sector of the target space. All maps from the base space $\Sigma$, must satisfy

$$
\begin{array}{lr}
\oint_{C_{i}} \mathrm{~d} X^{r}=2 \pi S_{i}^{r} R^{r}, & r=1,2, \\
\oint_{C_{i}} \mathrm{~d} X^{m}=0 & m=3, \ldots, 9,
\end{array}
$$


for $i=1,2$ and

$$
\int_{\Sigma} \mathrm{d} X^{r} \wedge \mathrm{d} X^{s}=\epsilon^{r s}\left(2 \pi^{2} R_{1} R_{2}\right) n,
$$

where $n=\operatorname{det} S_{i}^{r}$ is fixed, each entry $S_{i}^{r}$ is integer, and $R_{1}$ and $R_{2}$ denote the radii of the target component $S^{1} \times S^{1}$. Note that (3) describe maps from $\Sigma$ to $S^{1} \times S^{1}$ with $\mathrm{d} X^{m}$ exact one-forms and $\mathrm{d} X^{r}$ nontrivial closed one-forms. The only restriction upon these maps is the assumption that $n$ is fixed. The term on the left side of (4) describes the central charge of the supersymmetric algebra. As we shall see next, the factor $R_{1} R_{2}(2 \pi)^{2}$ is the area of $\Sigma$ in the induced metric.

The general map satisfying (3)-(4) can be constructed explicitly. Any closed one-form $\mathrm{d} X^{r}$ may be expressed as the sum of a harmonic and an exact form,

$$
\mathrm{d} X^{r}=L_{s}^{r} \mathrm{~d} \hat{X}^{s}+\delta_{s}^{r} \mathrm{~d} A_{s} \quad s, r=1,2
$$

where $L_{s}^{r}$ are real numbers and $\mathrm{d} \hat{X}$ is a canonical basis of harmonic one-forms over $\Sigma$. The term $\mathrm{d} \hat{X}^{s}, s=1,2$, is found by considering the (unique) holomorphic one-form $\omega$, normalised with respect to the elements of the homology basis $C_{i}$, defined by:

$$
\oint_{C_{1}} w=1, \quad \oint_{C_{2}} w=\Pi,
$$

where $\Pi$ is the period of $\omega$ in the basis given by $C_{i}$. By construction, the imaginary part of $\Pi$ is positive. Let [16]

$$
\omega=\mathrm{d} \widetilde{X}^{1}+i \mathrm{~d} \widetilde{X}^{2}
$$

and define

$$
\mathrm{d} \hat{X}^{r}=\left(M^{-1} \mathrm{~d} \tilde{X}\right)^{r},
$$

where the constant matrix $M$ is given by

$$
M=\left(\begin{array}{cc}
1 & \operatorname{Re} \Pi \\
0 & \operatorname{Im} \Pi
\end{array}\right)
$$

Then [16]

$$
\oint_{C_{i}} \mathrm{~d} \hat{X}^{r}=\delta_{i}^{r}
$$

and

$$
\int_{\Sigma} \mathrm{d} \hat{X}^{r} \wedge \mathrm{d} \hat{X}^{s}=\epsilon^{r s}
$$

If (3) is to be satisfied, necessarily

$$
L_{s}^{r}=2 \pi R^{r} S_{s}^{r} .
$$


Condition (44) implies

$$
S_{s}^{r} S_{u}^{t} \epsilon^{s u}=n \epsilon^{r t}
$$

Define the scalar density $\sqrt{W}$ by

$$
\epsilon^{a b} \partial_{a} \hat{X}^{r} \partial_{b} \hat{X}^{s} \epsilon_{r s}=\sqrt{W}
$$

where $\partial_{a} \equiv \partial / \partial \sigma^{a}, a=1,2, \sigma^{a}$ being local coordinates on $\Sigma$. Then

$$
\epsilon_{r s} \mathrm{~d} \hat{X}^{r} \wedge \mathrm{d} \hat{X}^{s}=\sqrt{W} \mathrm{~d} \sigma^{1} \wedge \mathrm{d} \sigma^{2}
$$

A change of the canonical basis of homology over $\Sigma$, implies varying the corresponding harmonic one-form $\mathrm{d} \hat{X}^{r} \rightsquigarrow T_{s}^{r} \mathrm{~d} \hat{X}^{s}$, where $T \in S L(2, Z)$, that is

$$
T_{s}^{r} T_{u}^{t} \epsilon^{s u}=\epsilon^{r t}
$$

$T_{s}^{r}$ integers. As the density $\sqrt{W}$ remains invariant under these transformations, they are area-preserving diffeomorphisms disconnected from the identity. The theory is then invariant under $S L(2, Z)$. The degrees of freedom are expressed in terms of $A_{r}$ and the discrete set of integers described by the harmonic one-forms. We can always fix these gauge transformations, within the conformal class of the target torus, by

$$
S_{i}^{r}=l^{r} \delta_{i}^{r}, \quad l^{1} l^{2}=n .
$$

Therefore

$$
\mathrm{d} X^{r}=2 \pi R^{r} l^{r} \mathrm{~d} \hat{X}^{r}+\delta_{s}^{r} \mathrm{~d} A_{s} .
$$

After the gauge fixing there is a residual invariance $\mathbb{Z}(2)$.

The complete expression for the Hamiltonian of the $D=11$ supermembrane subject to the topological conditions (3) and (4) turns out to be [14, 19, 12],

$$
\begin{aligned}
& H=\int \sqrt{W} \mathrm{~d} \sigma^{1} \wedge \mathrm{d} \sigma^{2}\left[\frac{1}{2}\left(\frac{P_{m}}{\sqrt{W}}\right)^{2}+\frac{1}{2}\left(\frac{\Pi^{r}}{\sqrt{W}}\right)^{2}+\frac{1}{4}\left\{X^{m}, X^{n}\right\}^{2}+\right. \\
& \left.\frac{1}{2}\left(\mathcal{D}_{r} X^{m}\right)^{2}+\frac{1}{4}\left(\mathcal{F}_{r s}\right)^{2}+\Lambda\left(\left\{P_{m}, X^{m}\right\}+\mathcal{D}_{r} \Pi^{r}\right)\right]+ \text { (fermionic term) }
\end{aligned}
$$

where [19, 12 ]

$$
\begin{array}{r}
\mathcal{D}_{r} X^{m}=D_{r} X^{m}+\left\{A_{r}, X^{m}\right\}, \\
\mathcal{D}_{r} \Pi^{r}=2 \pi R^{r} l^{r} \frac{\epsilon^{a b}}{\sqrt{W}} \partial_{a} \hat{X}^{r} \partial_{b}\left(\frac{\Pi^{r}}{\sqrt{W}}\right)+\left[A_{r}, \Pi^{r}\right], \\
\mathcal{F}_{r s}=D_{r} A_{s}-D_{s} A_{r}+\left[A_{r}, A_{s}\right]
\end{array}
$$

and $D_{r}=2 \pi R^{r} l^{r} \frac{\epsilon^{a b}}{\sqrt{W}} \partial_{a} \hat{X}^{r} \partial_{b}$. The associated mass operator is $(\text { mass })^{2}=$ 
$Z^{2}+H$, where $Z^{2}=\frac{1}{8}\left((2 \pi)^{2} n R_{1} R_{2}\right)^{2}$ is the central charge.

2.1. Semiclassical regime of the bosonic Hamiltonian. The semiclassical approximation of the theory is obtained by considering only the quadratic terms in the above expression for the Hamiltonian. Let

$$
\begin{aligned}
& H_{\mathrm{sc}}=\int \sqrt{W} \mathrm{~d} \sigma^{1} \wedge \mathrm{d} \sigma^{2}\left[\frac{1}{2}\left(\frac{P_{m}}{\sqrt{W}}\right)^{2}+\frac{1}{2}\left(\frac{\Pi^{r}}{\sqrt{W}}\right)^{2}+\right. \\
& \left.\left.\frac{1}{2}\left(D_{r} X^{m}\right)^{2}+\frac{1}{4}\left(\widehat{\mathcal{F}}_{r s}\right)^{2}+\Lambda D_{r} \Pi^{r}\right]+ \text { (fermionic terms }\right),
\end{aligned}
$$

where, in the semiclassical approximation,

$$
\widehat{\mathcal{F}}_{r s}=D_{r} A_{s}-D_{s} A_{r} .
$$

The general solution to the constraint $D_{r} \Pi^{r}=0$ is

$$
\Pi^{r}=\epsilon^{r s} 2 \pi R^{s} l^{s} \epsilon^{a b} \partial_{a} \hat{X}^{s} \partial_{b}\left(\frac{\Pi}{\sqrt{W}}\right),
$$

where $\Pi$ is a scalar density.

The kinetic term $\Pi^{r} \dot{A}_{r}$ may be rewritten, once integration by parts has been carried out, as $p \dot{q}$ where $p=\sqrt{W} \frac{1}{2} \epsilon^{r s} \mathcal{F}_{r s}$ and $q=\frac{\Pi}{\sqrt{W}}$. Thus

$$
\begin{aligned}
H=\int \mathrm{d} \sigma^{1} & \wedge \mathrm{d} \sigma^{2} \sqrt{W}\left[\frac{1}{2}\left(\frac{\Pi^{r}}{\sqrt{W}}\right)^{2}+\frac{1}{4}\left(\widehat{\mathcal{F}}_{r s}\right)^{2}+\Lambda D_{r} \Pi^{r}\right]= \\
& =\frac{1}{k} \int \mathrm{d} \sigma^{1} \wedge \mathrm{d} \sigma^{2} \sqrt{W}\left[\frac{1}{2}\left(\frac{p}{\sqrt{W}}\right)^{2}+\frac{1}{2}\left(D_{r} q\right)^{2}\right]
\end{aligned}
$$

which coincides with the contribution to the Hamiltonian of the transverse modes $X^{m}, m=3, \ldots, 9$.

The above shows that, from a gauge independent point of view, the complete bosonic Hamiltonian in the semiclassical approximation is

$$
H_{\mathrm{sc}}^{B}=\int \mathrm{d} \sigma^{1} \wedge \mathrm{d} \sigma^{2} \sqrt{W}\left[\frac{1}{2}\left(\frac{P^{M}}{\sqrt{W}}\right)^{2}+\frac{1}{2}\left(D_{r} X^{M}\right)^{2}\right]
$$

where $M=1, \ldots, 8$. If we now express $X^{M}$ and $P^{M} / \sqrt{W}$ in terms of a complete orthonormal basis of scalar symmetries over over $\Sigma$, we obtain

$$
\begin{aligned}
& X^{M}=X_{A}^{M}(\tau) \exp \left[2 \pi i\left(a_{r} \hat{X}^{r}\right)\right](\sigma), \\
& \frac{P^{M}}{\sqrt{W}}=\rho_{A}^{M}(\tau) \exp \left[2 \pi i\left(a_{r} \hat{X}^{r}\right)\right](\sigma),
\end{aligned}
$$


where $A=\left(a_{1}, a_{2}\right)$. Thus, the bosonic contribution in the semiclassical Hamiltonian takes the form

$$
H_{\mathrm{sc}}^{\mathrm{B}}=\frac{1}{2}\left[\left(\rho_{A}^{M}\right)^{2}+\omega_{A}^{2}\left(X_{A}^{m}\right)^{2}\right] .
$$

The spectrum of $H_{\mathrm{sc}}^{\mathrm{B}}$ is then characterised in the following fashion. For any finite subset $\Omega$ of $\mathbb{N} \times \mathbb{N}$ there is an eigenvalue

$$
\begin{gathered}
\lambda_{\Omega}=\sum_{A \in \Omega} \omega_{A}, \\
\omega_{A}=\pi^{2} \sqrt{\left(R^{1} l^{1} a_{2}\right)^{2}+\left(R^{2} l^{2} a_{1}\right)^{2}} .
\end{gathered}
$$

This expression coincides with the particular case considered in [47] where the semiclassical quantization of the supermembrane over a fixed background was considered. The model in [47] correspond to a gauge fixed version of the supermembrane with central charges in the semiclassical approximation. By virtue of (10), for any given energy level $E$, there only exists a finite number of eigenvalues of $H_{\mathrm{sc}}^{\mathrm{B}}$ below $E$. The spectrum is therefore discrete.

This is the expression of the eigenvalues when the zero point energy has been eliminated. It is automatically cancelled when the semiclassical supersymmetric Hamiltonian is considered. This property was first demonstrated in [47] and it coincides exactly with the one for the semiclassical supermembrane with central charges.

\section{Operator Bounds on the EXACt Bosonic Hamiltonian}

According to the results reported in [13], the bosonic regularised Hamiltonian of the $D=11$ supermembrane with central charge, $H_{N}^{B}$, relates to its semiclassical approximation, $H_{\mathrm{sc}, N}^{B}$, by means of the following operator inequality:

$$
H_{N}^{B} \geq C_{N} H_{\mathrm{sc}, N}^{B}
$$

Here $N$ denotes the size of the truncation in the Fourier basis of $\Sigma$ and $C_{N}$ is a positive constant dependent on the size of the truncation. A crucial step in the proof of (11), relies heavily on the compactness of the unit ball of the configuration space which happens to be finite dimensional. In this section we show that the same operator relation holds true for the exact bosonic Hamiltonians, i.e. the case $N=\infty$, see Theorem 1. The main source of difficulties in the proof of this assertion lies in the fact that now the unit ball of the configuration space does not possess the property of being compact. We overcome these difficulties by carrying out a detailed analysis of each term involved in the expansion of the potential term of $H^{B}$. 
3.1. The configuration space and the gauge fixing condition. We firstly construct the configuration space for the supermembrane with central charges in a rigorous fashion and prescribe suitable gauge fixing condition.

Constant functions are harmonic, therefore the constant modes of the fields $X^{m}$ and $A_{r}$ belong to the harmonic sector in (5). In addition only derivatives of $X^{m}$ and $A_{r}$ appear in all the expressions. Let $\mathcal{H}^{1}$ denote the Hilbert space obtained by completing $C^{1}(\Sigma)$, modulo locally constant functions, with respect to the norm

$$
\|u\|^{2}=\int \mathrm{d}^{2} \sigma \sqrt{W} g^{a b} \partial_{a} u \partial_{b} \bar{u},
$$

where $g^{a b}$ is the inverse of the metric $g_{a b}=\partial_{a} \widehat{X}^{r} \partial_{b} \widehat{X}^{r}$ induced over $\Sigma$ by the minimal immersion $\widehat{X}_{r}$. Below we employ the following convention: for a field $u$,

$$
\langle u\rangle=\int_{\Sigma} \mathrm{d}^{2} \sigma \sqrt{W} u .
$$

For simplicity we will consider below only the case $n=1$ and $R_{1}=$ $R_{2}=1$.

Note that

$$
\begin{aligned}
D_{r} u D_{r} \bar{u} & =\frac{\epsilon^{a b}}{\sqrt{W}} \partial_{a} \widehat{X}^{r} \partial_{c} u \frac{\epsilon^{b d}}{\sqrt{W}} \partial_{b} \widehat{X}^{r} \partial_{d} \bar{u} \\
& =g^{c d} \partial_{c} u \partial_{d} \bar{u}
\end{aligned}
$$

and

$$
\{u, w\} \equiv \frac{\epsilon^{a b}}{\sqrt{w}} \partial_{a} u \partial_{b} w=\epsilon^{r s} D_{r} u D_{s} w
$$

so that

$$
\|u\|^{2}=\left\langle D_{r} u D_{r} \bar{u}\right\rangle
$$

Let

$$
\|u\|_{4,2}=\left(\left\langle D_{r} u\right\rangle^{2}+\left\langle D_{r} D_{s} u\right\rangle^{2}\right)^{1 / 4} .
$$

Below and elsewhere $X^{m}, A_{r}$ are assumed to be members of the configuration space $\mathcal{H}^{4,2}$ of fields $u \in \mathcal{H}^{1}$ such that $\|u\|_{4,2}<\infty$. The left hand side of (12) is a well defined norm in $\mathcal{H}^{4,2}$. The latter is a linear space, however we do not make any assumption about completeness.

The potential, $V$, of the bosonic sector of the supermembrane with central charges is well defined in $\mathcal{H}^{4,2}$ as

$$
V=\left\langle\mathcal{D}_{r} X^{m} \mathcal{D}_{r} X^{m}+\frac{1}{4} \mathcal{F}_{r s} \mathcal{F}_{r s}\right\rangle .
$$


The introduction of the constrained space $\mathcal{H}^{4,2} \subset \mathcal{H}^{1}$ is justified by the fact that homogeneous terms of order 4 are present on the right hand side of (13), so $V$ is not well defined in the whole space $\mathcal{H}^{1}$.

The following gauge fixing conditions are equivalent to those considered in [12, 13, 14],

$$
\begin{aligned}
& D_{1} A_{1}=0 \\
& \text { if } D_{1} A_{2}=0 \text { then } A_{2}=0 .
\end{aligned}
$$

These are obtained by expressing the fields in terms of an orthonormal basis over $\Sigma$. Integration by parts yields $\left\langle D_{2} A_{1} D_{1} A_{2}\right\rangle=0$. Similarly we also have $\left\langle D_{2} A_{1}\left\{A_{1}, A_{2}\right\}\right\rangle=0$. Note that $\left\langle\left(D_{1} A_{2}\right)^{2}\right\rangle=0$, implies $A_{2}=0$.

\subsection{The uniform quadratic bound for the bosonic potential.}

Let $\rho^{2}$ be the potential term of $H_{\mathrm{sc}}^{B}$, so that

$$
\rho^{2}=\left\langle D_{r} X^{m} D_{r} X^{m}+\left(D_{1} A_{2}\right)^{2}+\left(D_{2} A_{1}\right)^{2}\right\rangle .
$$

We may rewrite

$$
V=\rho^{2}+2 \mathbf{B}+\mathbf{A}^{2}
$$

where

$$
\begin{gathered}
\mathbf{B}=\left\langle D_{r} X^{m}\left\{A_{r}, X^{m}\right\}+D_{1} A_{2}\left\{A_{1}, A_{2}\right\}\right\rangle, \\
\mathbf{A}^{2}=\left\langle\left\{A_{1}, X^{m}\right\}^{2}+\left\{A_{2}, X^{m}\right\}^{2}+\left\{A_{1}, A_{2}\right\}^{2}+\left\{X^{m}, X^{n}\right\}^{2}\right\rangle .
\end{gathered}
$$

Theorem 1. There exists a constant $0<C \leq 1$, such that

$$
V \geq C \rho^{2}, \quad \forall X^{m}, A_{r} \in \mathcal{H}^{4,2} .
$$

The remaining parts of this section is devoted to showing the validity of Theorem 1.

By definition,

$$
V=\rho^{2}\left(1+\frac{2 \mathbf{B}}{\rho^{2}}+\frac{\mathbf{A}^{2}}{\rho^{2}}\right)=\rho^{2}\left(1+2 b R+a^{2} R^{2}\right),
$$

where

$$
\begin{gathered}
R=\left\|\left(X^{m}, A_{r}\right)\right\|_{4,2}, \\
a^{2}=\frac{\mathbf{A}^{2}}{R^{2} \rho^{2}} \quad \text { and } \quad b=\frac{\mathbf{B}}{\rho^{2} R} .
\end{gathered}
$$

Since both terms $a^{2}$ and $b$ are homogeneous in $X^{m}$ and $A_{r}$, they are constant in $R$. Without loss of generality we assume that $a^{2}$ and $b$ are always evaluated at fields $X^{m}, A_{r}$, normalised by the condition $R=1$.

Let

$$
P(R)=1+2 b R+a^{2} R^{2}
$$


be the real polynomial whose variable is $R \geq 0$. The existence of a constant $C>0$ satisfying (15) is equivalent to the condition

$$
\inf _{\left\|\left(X^{m}, A_{r}\right)\right\|_{4,2}=1}\left[\inf _{R \geq 0} P(R)\right] \geq C .
$$

Note that $\mathbf{B}$ is the inner product in $\mathcal{H}_{1}$ of the field

$$
\left(D_{1} X^{m}, D_{2} X^{m}, D_{1} A_{2}, 0\right)
$$

and the field

$$
\left(\left\{A_{1}, X^{m}\right\},\left\{A_{2}, X^{m}\right\},\left\{A_{1}, A_{2}\right\},\left\{X^{m}, X^{n}\right\}\right),
$$

while $\mathbf{A}$ is the norm of the latter. Thus $\mathbf{A}^{2}=0$ yields $\mathbf{B}=0$, so the condition $a^{2}=0$ implies $P(R)=1$. Hence

$$
\begin{aligned}
\inf _{\left\|\left(X^{m}, A_{r}\right)\right\|_{4,2}=1}\left[\inf _{R \geq 0} P(R)\right] & =\min \left[1, \inf _{a \neq 0}\left(1-\frac{b^{2}}{a^{2}}\right)\right] \\
& =\inf _{a \neq 0}\left(1-\frac{b^{2}}{a^{2}}\right)
\end{aligned}
$$

in (17).

The validity of the following lemma will immediately ensure (17), hence Theorem 1 .

Lemma 2. Let $a$ and $b$ be the quantities defined by (16). Then

$$
\inf _{a \neq 0}\left(1-\frac{b^{2}}{a^{2}}\right)>0
$$

Proof. We proceed by contradiction. Suppose that

$$
\inf _{a \neq 0}\left(1-\frac{b^{2}}{a^{2}}\right)=0 .
$$

Then we can find sequences $\left(X^{m}\right)_{j},\left(A_{r}\right)_{j} \in \mathcal{H}^{4,2}$, such that $\left\|\left(\left(X^{m}\right)_{j},\left(A_{r}\right)_{j}\right)\right\|_{4,2}=$ 1 and

$$
\frac{b_{j}^{2}}{a_{j}^{2}} \rightarrow 1
$$

as $j \rightarrow \infty$, where $a_{j}, b_{j}$ are the quantities defined by (16) for the fields at the $j$ th place of the sequences.

By construction

$$
\frac{b^{2}}{a^{2}}=\frac{\left\langle D_{r} X^{m}\left\{A_{r}, X^{m}\right\}+D_{1} A_{2}\left\{A_{1}, A_{2}\right\}+\left(D_{2} A_{1}\right) 0+0\left\{X^{m}, X^{n}\right\}\right\rangle^{2}}{\rho^{2} \mathbf{A}^{2}} .
$$

Thus, for each $j$, the left hand side of (18) is the inner product of two unit vectors in $\mathcal{H}^{1}$. By virtue of the Cauchy Schwarz inequality, these two vectors should become increasingly parallel as as $j \rightarrow \infty$. Since the 
quantities $a^{2}$ and $b^{2}$ remain constant if we multiply the field $\left(X^{m}, A_{r}\right)$ by a constant, without loss of generality we can chose our sequences $\left(X^{m}\right)_{j},\left(A_{r}\right)_{j}$, such that

$$
\begin{gathered}
\left\langle\left(\frac{D_{r}\left(X^{m}\right)_{j}}{\rho_{j}}-\frac{\left\{\left(A_{r}\right)_{j},\left(X^{m}\right)_{j}\right\}}{\mathbf{A}_{j}}\right)^{2}\right\rangle \rightarrow 0 \\
\left\langle\left(\frac{D_{1}\left(A_{2}\right)_{j}}{\rho_{j}}-\frac{\left\{\left(A_{1}\right)_{j},\left(A_{2}\right)_{j}\right\}}{\mathbf{A}_{j}}\right)^{2}\right\rangle \rightarrow 0, \\
\left\langle\left(\frac{D_{2}\left(A_{1}\right)_{j}}{\rho_{j}}\right)^{2}\right\rangle \rightarrow 0 \text { and }\left\langle\left(\frac{\left\{\left(X^{m}\right)_{j},\left(X^{n}\right)_{j}\right\}}{\mathbf{A}_{j}}\right)^{2}\right\rangle \rightarrow 0 .
\end{gathered}
$$

When $r=1$, the left side of (19), is

$$
\begin{aligned}
\left\langle\left(\frac{D_{1}\left(X^{m}\right)_{j}}{\rho_{j}}\right.\right. & \left.\left.-\frac{D_{2}\left(A_{1}\right)_{j} D_{1}\left(X^{m}\right)_{j}}{\mathbf{A}_{j}}\right)^{2}\right\rangle \geq \\
& \left\langle\left(\frac{D_{1}\left(X^{m}\right)_{j}}{\rho_{j}}\right)^{2}\right\rangle-2\left|\left\langle\frac{D_{1}\left(X^{m}\right)_{j}}{\rho_{j}} \frac{D_{2}\left(A_{1}\right)_{j} D_{1}\left(X^{m}\right)_{j}}{\mathbf{A}_{j}}\right\rangle\right| .
\end{aligned}
$$

By virtue of the Cauchy Schwarz inequality,

$$
\begin{aligned}
\mid\left\langle\frac{D_{1}\left(X^{m}\right)_{j}}{\rho_{j}}\right. & \left.\frac{D_{2}\left(A_{1}\right)_{j} D_{1}\left(X^{m}\right)_{j}}{\mathbf{A}_{j}}\right\rangle|=|\left\langle\left(D_{1}\left(X^{m}\right)_{j}\right)^{2} \frac{D_{2}\left(A_{1}\right)_{j}}{\rho_{j} \mathbf{A}_{j}}\right\rangle \mid \\
& \leq\left\langle\left(D_{1}\left(X^{m}\right)_{j}\right)^{4}\right\rangle^{1 / 2}\left\langle\left(\frac{D_{2}\left(A_{1}\right)_{j}}{\rho_{j}}\right)^{2}\right\rangle^{1 / 2} \frac{1}{\mathbf{A}_{j}} \\
& \leq\left\langle\left(\frac{D_{2}\left(A_{1}\right)_{j}}{\rho_{j}}\right)^{2}\right\rangle^{1 / 2} \frac{1}{\mathbf{A}_{j}} .
\end{aligned}
$$

Furthermore, analogous results also hold true for the left sides of (19) when $r=2$ and the left side of (20). Hence, if $\mathbf{A}_{j} \not \rightarrow 0$, the above, along with (19) and (20), imply

$$
\frac{\left\langle\left(D_{r}\left(X^{m}\right)_{j}\right)^{2}+\left(D_{1}\left(A_{2}\right)_{j}\right)^{2}+\left(D_{2}\left(A_{1}\right)_{j}\right)^{2}\right\rangle}{\rho_{j}^{2}} \rightarrow 0
$$

which is impossible.

It is only left showing that the case $\mathbf{A}_{j} \rightarrow 0$ also leads to a contradiction. We proceed as follows.

Let $\Delta$ denote the Laplacian operator acting on $L^{2}(\Sigma)$. Integration by parts yields

$$
\left\langle D_{r}\left(D_{1}\left(X^{m}\right)_{j}\right)^{2} D_{r}\left(D_{1}\left(X^{m}\right)_{j}\right)^{2}\right\rangle=\left\langle\left[(-\Delta)^{1 / 2}\left(D_{1}\left(X^{m}\right)_{j}\right)^{2}\right]^{2}\right\rangle .
$$


Then, $\left(W^{m}\right)_{j}=(-\Delta)^{1 / 2}\left(D_{1}\left(X^{m}\right)_{j}\right)^{2} \in L^{2}(\Sigma)$. Since $\left\|\left(X^{m}\right)_{j}\right\|_{4,2} \leq 1$, $\left\|\left(W^{m}\right)_{j}\right\|_{L^{2}} \leq 1$. Now $(-\Delta)^{-1 / 2}$ is a compact operator and

$$
\left(D_{1}\left(X^{m}\right)_{j}\right)^{2}=(-\Delta)^{-1 / 2}\left(W^{m}\right)_{j}
$$

Thus $\left(D_{1}\left(X^{m}\right)_{j}\right)^{2}$ has a subsequence which is convergent in $\|\cdot\|_{L^{2}}$ to an accumulation point, say $Y_{1}^{m} \in L^{2}(\Sigma)$.

Similarly $\left(D_{2}\left(X^{m}\right)_{j}\right)^{2},\left(D_{1}\left(A_{2}\right)_{j}\right)^{2}$ and $\left(D_{2}\left(A_{1}\right)_{j}\right)^{2}$ have corresponding subsequences, convergent in $\|\cdot\|_{L^{2}}$ to accumulation points, $Y_{2}^{m}, Z_{1}$ and $Z_{2}$ in $L^{2}(\Sigma)$. Furthermore $Y_{r}^{m}$ and $Z_{r}$ lie on $L^{4}(\Sigma)$, so that we can evaluate $P(R)$ at $\left(\left(Y_{r}^{m}\right)^{1 / 2},\left(Z_{r}\right)^{1 / 2}\right)$. As $\mathbf{A}^{2}=0$ when we evaluate at these limit fields, in fact $P(R)$ achieves the constant value 1 . But, since $b$ and $a^{2}$ are continuous in $\left(D_{r} X^{m}, D_{1} A_{2}, D_{2} A_{1}\right)$ for the norm $\|\cdot\|_{L^{2}}$, this contradicts the condition $b_{j}^{2} / a_{j}^{2} \rightarrow 1$. The proof of the lemma is hence completed.

In order to define rigorously the bosonic Hamiltonian in the noncompact infinite dimensional configuration space $\mathcal{H}^{4,2}$, the operator is expressed as

$$
H^{B}=\left[V_{\text {quartic }}+V_{\text {cubic }}+(1-C) V_{\text {quadratic }}\right]+\left[-\Delta+C V_{\text {quadratic }}\right] .
$$

The term inside the first bracket acts as a multiplication operator on the Hilbert space of states. The one inside the second bracket may be expressed in terms of creation and annihilation operators.

By virtue of Theorem 1,

$$
H^{B} \geq-\Delta+C V_{\text {quadratic }}
$$

The infinite zero point energy can be extracted from the same operator at both sides of this inequality. This zero point energy will be automatically cancelled out when we considered the supersymmetric theory. According to the results of [14] and [15], the fermionic contribution does not change the qualitatively properties of the bosonic Hamiltonian of the regularised model. We expect this to be also the case for the exact model.

Identity (22) alongside with (10) implies that the spectrum of the exact theory is discrete with finite multiplicities. As we mentioned above, the same inequality was demonstrated to be valid for the regularised bosonic model. 


\section{ON THE LARGE $N$ LIMIT OF THE SEMICLASSICAL BOSONIC} HAMILTONIAN

The semiclassical Hamiltonian of the regularised model is,

$$
\begin{aligned}
H_{\mathrm{sc}, N} & =\operatorname{Tr}\left[\frac{1}{2 N^{3}}\left(\left(P^{m}\right)^{2}+\left(\Pi_{r}\right)^{2}\right)+\frac{n^{2}}{8 \pi^{2} N^{3}}\left(\frac{i}{N}\left[T_{V_{r}}, X^{m}\right] T_{-V_{r}}\right)^{2}\right. \\
& +\frac{n^{2}}{16 \pi^{2} N^{3}}\left(\frac{i}{N}\right)^{2}\left(\left[T_{V_{r}}, A_{r}\right] T_{-V_{s}}-\left[T_{V_{s}}, A_{r}\right] T_{-V_{r}}\right)^{2} \\
& \left.-\frac{i}{N}\left(\frac{i n}{4 \pi N^{3}}\right) \bar{\Psi} \gamma_{-} \gamma_{r}\left[T_{V_{r}}, \Psi\right] T_{-V_{r}}\right]
\end{aligned}
$$

Here the $S U(N)$ matrices $T_{A}=N \omega^{\frac{1}{2} a_{1} a_{2}} P^{a_{1}} Q^{a_{2}}, T_{0}=N \mathbb{I}, A=\left(a_{1}, a_{2}\right)$ and $P, Q$ are the Heisenberg matrices satisfying the Weyl condition $P Q=\omega Q P$ where $\omega=e^{\frac{2 \pi i}{N}}$. The generators of the algebra of $S U(N)$ may be expressed in terms of $T_{A}$. The fields may be expanded in this basis as $X^{m}=X^{m A} T_{A}$, satisfying

$$
\begin{aligned}
& {\left[T_{V_{r}}, T_{A}\right]=f_{V_{r} A}^{V_{r}+A} T_{V_{r}+A}} \\
& T_{A} T_{B}=N \omega^{\frac{1}{2} A \wedge B} T_{A+B} .
\end{aligned}
$$

Here $f_{A B}^{C}=2 i N \sin \left(\frac{(A \wedge B) \pi}{N}\right) \delta(A+B-C)$ are the standard structure constants for the regularisation via $S U(N)$ of a two-torus, $A \wedge B=$ $a_{1} b_{2}-a_{2} b_{1}, V_{1}=(1,0)$ and $V_{2}=(0,1)$.

The bosonic contribution in $H_{\mathrm{sc}, N}$ consists of two components, one related to the transverse field sector and the other related to the induced gauge fields on the world-volume of the membrane.

A direct calculation shows that the transverse field contribution,

$$
\operatorname{Tr}\left(\frac{n^{2}}{8 \pi^{2} N^{3}}\left(\frac{i}{N}\left[T_{V_{r}}, X^{m}\right] T_{-V_{r}}\right)^{2}\right),
$$

is equivalent to

$$
V_{\mathrm{sc}_{1}, N}=\sum_{A}\left|X^{m A}\right|^{2} w_{A, N}^{2}
$$

where

$$
w_{A, N}=\frac{n N}{\sqrt{2} \pi} \sqrt{\left(\sin \left(\frac{a_{1} \pi}{N}\right)\right)^{2}+\left(\sin \left(\frac{a_{2} \pi}{N}\right)\right)^{2}} .
$$

The second contribution corresponds to the gauge fields defined on the world volume of the membrane as a result of the central charges 
induced by the winding. This contribution is

$$
V_{\mathrm{sc}_{2}, N}=\frac{n^{2}}{8 \pi^{2} N^{5}} \operatorname{Tr}\left(\left(\left[T_{V_{1}}, A_{2}\right] T_{-V_{1}}-\left[T_{V_{2}}, A_{1}\right] T_{-V_{2}}\right)^{2}\right) .
$$

Here we fix the remaining gauge fields by imposing the following constraint

$$
A_{1}^{(m, n)}=0 \quad \text { for } \quad n \neq 0, \quad A_{2}^{(p, q)}=0 \quad \text { for } \quad q=0 .
$$

Then

$$
\left[T_{V_{1}}, A_{2}\right] T_{-V_{1}}=N \sum_{B} f_{V_{1} B}^{V_{1}+B} \omega^{+\frac{1}{2} b_{2}} T_{B}
$$

for $r=1$ and a similar identity holds true for $r=2$. After performing the canonical reduction associated to the above gauge fixing conditions, we obtain

$$
V_{\mathrm{sc}_{2}, N}=\sum_{B} A_{1}^{B} \overline{A_{1}^{B}}\left(w_{1, B, N}\right)^{2}+A_{2}^{B} \overline{A_{2}^{B}}\left(w_{2, B, N}\right)^{2},
$$

where

$$
\begin{aligned}
& w_{1, B, N}=\frac{n N}{\sqrt{2 \pi}} \sin \left(\frac{b_{1} \pi}{N}\right) \text { and } \\
& w_{2, B, N}=\frac{n N}{\sqrt{2 \pi}} \sqrt{\sin \left(\frac{b_{1} \pi}{N}\right)^{2}+\sin \left(\frac{b_{2} \pi}{N}\right)^{2}} .
\end{aligned}
$$

These coefficients correspond to contributions as for the $X^{m}$ modes.

By virtue of (23) and (25), the bosonic regularised semiclassical Hamiltonian realises as

$$
H_{\mathrm{sc}, N}^{\mathrm{B}}=-\Delta_{\left(X^{m A}, A_{1}^{B}, A_{2}^{B}\right)}+V_{\mathrm{sc}_{1}, N}+V_{\mathrm{sc}_{1}, N}+c_{N},
$$

a quantum mechanical harmonic oscillator acting on the Hilbert space $L^{2}\left(\left(X^{m A}, A_{1}^{B}, A_{2}^{B}\right) \in \mathbb{R}^{\Lambda}\right)$. Here $N$ is a large parameter representing the number of $D 0$ branes in the regularisation process; $\left(X^{m A}, A_{1}^{B}, A_{2}^{B}\right) \in$ $\mathbb{R}^{\Lambda}$ lies in the space coordinates and $\Lambda=8\left(N^{2}-1\right)$ for an $S U(N)$ regularisation. Here $A=(m, n)$ and $B=(p, q)$. The constant $c_{N}$ is a shift in the position of the ground state energy and it is chosen, so that the ground energy of $H_{\mathrm{sc}, N}^{\mathrm{B}}$ is exactly zero.

This characterisation of $H_{\mathrm{sc}, N}^{\mathrm{B}}$ as an elliptic partial differential operator is convenient for determining spectral [14] and heat kernel [15] properties of the Hamiltonian of the supermembrane, once the regularisation process has been carried out. We now consider this representation in order to study the large $N$ limit of $H_{\mathrm{sc}, N}^{\mathrm{B}}$ and its connection with $H_{\mathrm{sc}}^{\mathrm{B}}$. All limiting process below refer to taking $N \rightarrow \infty$. 
Lemma 3. Each eigenvalue of $H_{\mathrm{sc}, N}^{B}$ converges to a corresponding eigenvalue of $H_{\mathrm{sc}}^{B}$ as $N \rightarrow \infty$.

The large $N$ limit is defined by taking a fixed energy $E$ and then comparing the spectrum of the operators below $E$ in the limit $N \rightarrow \infty$.

Proof. Firstly note that there is a one to one correspondence between individual excited state eigenvalues of $H_{\mathrm{sc}, \mathrm{N}}^{B}$ and $H_{\mathrm{sc}}^{B}$, and finite subsets of $\mathbb{N} \times \mathbb{N}$. When $N<\infty$, the variables in (27) can be separated, so the eigenfunctions of $H_{\mathrm{sc}, N}^{\mathrm{B}}$ are given explicitly in terms of creation operators as,

$$
a_{C_{1}}^{(N) \dagger} \ldots a_{C_{\Lambda}}^{(N) \dagger}|0\rangle
$$

with associated eigenvalue

$$
\lambda_{\mathcal{F}, N}=\omega_{C_{1}, N}+\cdots+\omega_{C_{\Lambda}, N}
$$

corresponding to the set

$$
\mathcal{F}=\left\{C_{1}, \ldots, C_{\Lambda}\right\}
$$

Here $\omega_{C, N}$ are either $w_{A, N}, w_{1, B, N}$ or $w_{2, B, N}$. This provides an indexing for the spectrum of $H_{\mathrm{sc}, N}^{\mathrm{B}}$ in terms of finite subsets of $\mathbb{N} \times \mathbb{N}$ with at most $\Lambda$ elements. Similarly, for the case of the limiting $H_{\mathrm{sc}}^{\mathrm{B}}$, the eigenfunctions are constructed in terms of creation operators, but now the sequences can be of arbitrary length. Thus, the eigenvalues are in one to one correspondence now with all finite subsets of $\mathbb{N} \times \mathbb{N}$.

For any given finite subset $\mathcal{F}$ of $\mathbb{N} \times \mathbb{N}$, we just have to choose $N$ larger than the number of elements of $\mathcal{F}$, in order to ensure that $\mathcal{F}$ is also included in the indexing for the eigenvalues of $H_{\mathrm{sc}, N}^{B}$. As $\mathcal{F}$ is finite, $\omega_{C, N} \rightarrow \omega_{C}$ and the expressions for the eigenvalues are finite sums,

$$
\lambda_{\mathcal{F}, N} \rightarrow \lambda_{\mathcal{F}}
$$

as required.

The following remark on the multiplicity of the spectrum of $H_{\mathrm{sc}}^{B}$ is in place. For a given index $A=(m, n)$, see (10),

$$
\omega_{A} \geq \pi^{2} \min \left\{R^{1} l^{1}, R^{2} l^{2}\right\},
$$

where the constants on the right hand side are independent of $A$. Then, for a given finite subset $\mathcal{F}$ with $\Phi$ elements,

$$
\lambda_{\mathcal{F}} \geq \Phi \pi^{2} \min \left\{R^{1} l^{1}, R^{2} l^{2}\right\} .
$$

Hence, the class of subsets $\tilde{\mathcal{F}}$ such that $\lambda_{\mathcal{F}}=\lambda_{\tilde{\mathcal{F}}}$, is limited by the fact that $\tilde{\mathcal{F}}$ can not have more than $\lambda_{\mathcal{F}} / \pi^{2} \min \left\{R^{1} l^{1}, R^{2} l^{2}\right\}$ elements. This ensures that each eigenvalue of $H_{\mathrm{sc}}^{B}$ is of finite multiplicity. 
The above lemma shows that the spectra of $H_{\mathrm{sc}, N}^{\mathrm{B}}$ converge to the spectrum of $H_{\mathrm{sc}}^{\mathrm{B}}$. However it does not provide information about the large $N$ limit of $H_{\mathrm{sc}, N}^{B}$ and its relation with $H_{\mathrm{sc}}^{B}$.

The Hamiltonian $H_{\mathrm{sc}}^{B}$ is equivalent, under unitary transformation, to a self-adjoint operator acting on the Hilbert space $L^{2}\left(\ell_{2}, \mathrm{~d} \gamma\right)$, where $\mathrm{d} \gamma$ is a Gaussian measure on $\ell_{2}$. Recall that $\ell_{2}$ comprises square summable $\left(Y^{C}\right)_{C \in \mathbb{N} \times \mathbb{N}}$ such that $\|Y\|^{2}=\sum_{C}\left|Y^{C}\right|^{2}<\infty$. A procedure for constructing Gaussian measures in $\ell_{2}$ is described in the monograph [34].

For each wave function $\psi \in L^{2}\left(\ell_{2}, \mathrm{~d} \gamma\right)$, there exists $\psi_{N} \in L^{2}\left(\mathbb{R}^{\Lambda}, \mathrm{d} \gamma^{\Lambda}\right)$ such that $\psi_{N} \rightarrow \psi$. Here $\mathrm{d} \gamma^{\Lambda}=e^{-\|Y\|^{2} / 2} \mathrm{~d} Y$ is the standard Gaussian measure in $\mathbb{R}^{\Lambda}$. By performing a suitable change of coordinates, the Hamiltonian $H_{\mathrm{sc}, N}^{\mathrm{B}}$ is also an operator acting on $L^{2}\left(\mathbb{R}^{\Lambda}, \mathrm{d} \gamma^{\Lambda}\right)$. Hence, we are in the position of being able to compare the exact model with the regularised one. Indeed, since $L^{2}\left(\mathbb{R}^{\Lambda}, \mathrm{d} \gamma^{\Lambda}\right)$ are subspaces of $L^{2}\left(\ell_{2}, \mathrm{~d} \gamma\right)$ via the natural identification

$$
\phi\left(\left(Y^{C}\right)\right) \longmapsto \phi\left(\left(Y^{C}\right), 0, \ldots\right), \quad\left(Y^{C}\right) \in \mathbb{R}^{\Lambda},
$$

both operators $H_{\mathrm{sc}, N}^{\mathrm{B}}$ and $H_{\mathrm{sc}}^{\mathrm{B}}$ act in the same subspace. Note that here we must use the fact that $\mathrm{d} \gamma$ is Gaussian in order to ensure that the right hand side is a member of the latter space. In the other direction, we have the projected states

$$
\phi\left(Y^{(1,0)}, \ldots\right) \longmapsto \phi\left(\left(Y^{C}\right), 0, \ldots\right)=: \phi_{N}\left(Y^{(1,0)}, \ldots\right) .
$$

for all $\phi\left(Y^{(1,0)}, \ldots\right) \in L^{2}\left(\ell_{2}, \mathrm{~d} \gamma\right)$. This identification gives a precise meaning to the limit

$$
\lim _{N \rightarrow \infty}\left\langle\psi,\left(H_{\mathrm{sc}, N}^{\mathrm{B}} \phi_{N}-H_{\mathrm{sc}}^{\mathrm{B}} \phi\right)\right\rangle,
$$

making it possible to verify rigorously whether $H_{\mathrm{sc}, N}^{\mathrm{B}} \rightarrow H_{\mathrm{sc}}^{\mathrm{B}}$ in the weak topology.

For given initial states $\phi, \psi \in L^{2}\left(\ell_{2}, \mathrm{~d} \gamma\right)$ and $t>0$, we can also compute the limit

$$
\lim _{N \rightarrow \infty}\left\langle\phi_{N}, e^{-H_{\mathrm{sc}, N}^{\mathrm{B}} t} \psi_{N}\right\rangle
$$

via the Mehler formula. For this we should recall that the heat kernel of the regularised semiclassical Hamiltonian can be found explicitly, $e^{-H_{\mathrm{sc}, N}^{\mathrm{B}} t}$ is the Ornstein-Uhlenbeck semigroup. This rises the question of whether the exact semigroup $e^{-H_{\mathrm{sc}}^{\mathrm{B}} t}$ could possibly be characterised using the Feynman-Kac formula.

Note that the characterisation of the regularised Hamiltonian in the space with Gaussian measure is far more advantageous than our previous approach of using the space with Lebesgue measure. Indeed one 
can easily prove that it is not possible to construct a Lebesgue measure in $\ell_{2}$.

\section{Centre of the group, mass gap And Confinement}

In the first part of this paper we have discussed spectral properties of the supermembrane with central charges. Let us now investigate its behaviour, or in equivalent manner, the behaviour of the symplectic NCSYM theory, at extreme energies. This case corresponds to analysing the supermembrane with central charges for $g=3$, that is compactified on $T^{6}$, and performing a further compactification of the fifth coordinate to $S^{1}$. The spectral properties discussed above remain invariant and the theory corresponds to an $N=1$ NCSYM of dimension $2+1$ on a $4-D$ target-space.

By virtue of the results of [35] and [36], a permanent quark confinement occurs in a gauge theory, if its vacuum condenses into a state which resembles a superconductor. This proposal consists in taking the confinement of quarks as dual to the Meissner effect, where the roles of magnetism and electricity is interchanged. In this approach, a non-Abelian gauge theory is considered in terms of an Abelian theory enriched with Dirac magnetic monopoles. As we will see next, this is in correspondence with the situation we are presently discussing in the case of the 4-D target space, under the assumption that the underlying $N=1$ Yang-Mills theory is symplectic and non-commutative.

Our first task will be to study the symmetries of the theory. We will then identify the centre of the group relative to the residual symmetry. This latter symmetry plays a role in the creation of confinement and, through its breaking, the theory enters in a quark-gluon plasma phase corresponding to the supermembrane without central charges.

5.1. Symmetries. The $D=11$ supermembrane in the light cone gauge with a Minkowski target space possesses a residual invariance, associated to the infinite group of area preserving diffeomorphisms $\operatorname{Diff}^{\infty}(\Sigma)$ on a Riemann surface $\Sigma$ of genus $g$, [3]. The first class constraints of the theory generates area preserving diffeomorphisms homotopic to the identity, $\operatorname{Diff}_{\mathbb{I}}^{\infty}(\Sigma)$. The local term generates area preserving diffeomorphisms associated to exact 1-forms, [3]:

$$
\partial_{r}\left(\sqrt{\omega(\sigma)} \xi^{r s}(\sigma, \tau)\right) \equiv D_{r} \xi^{r}(\sigma)=0 ; \quad \xi^{r}(\sigma)=\frac{\epsilon^{r s}}{\sqrt{\omega(\sigma)}} \partial_{s} \xi(\sigma)
$$


The global term, on the other hand, generates area preserving diffeomorphisms associated to harmonic one-forms. The local constraint corresponds to the Gauss law for the non-commutative symplectic YangMills formulation. We might call it, the generator of gauge transformations.

Let us analyse the diffeomorphisms generated by the global constraint. Since only derivatives terms are present, the harmonic functions on the theory may be defined up to a constant. Thus, the global constraint generates an abelian sub-group of diffeomorphisms. In fact, from the considerations of Section 2, we obtain

$$
\left\{\xi_{1}, \xi_{2}\right\}=(\text { constant })
$$

where $\xi_{1}$ and $\xi_{2}$ are harmonic parameters. Hence the commutator of two such a diffeomorphisms is in the harmonic class of zero. In addition, each harmonic one-form may be expanded in terms of the basis $\mathrm{d} \widehat{X}^{r}$ as

$$
\mathrm{d} \xi=a_{1} \mathrm{~d} \widehat{X}^{1}+a_{2} \mathrm{~d} \widehat{X}^{2}
$$

where $a_{1}$ and $a_{2}$ are real numbers. The algebra of infinitesimal diffeomorphisms generated by the global constraint contains then a realisation of $u(1) \times u(1)$. This symmetry of the supermembrane with central charges preserves gauge equivalent classes. The present interpretation of this new symmetry is completely different from the approach followed in [9], where a $S U(N)$ regularisation of the supermembrane on a compactified target space was discussed. Moreover, since a consistent $S U(N)$ regularisation of the supermembrane with central charges was found in [12], this symmetry should reduce to $\mathbb{Z}_{N} \times \mathbb{Z}_{N}$ in the regularised formulation.

The presence of $r$ closed, but not exact, forms can be regarded in the dual picture, i.e. in the 10-D IIA description, as the existence of $r$ $U(1)$ gauge fields corresponding to the compactification process. This means that the compactified supermembrane has the following gauge symmetries from the type IIA point of view,

$$
\operatorname{Diff}_{\mathbb{I}}^{\infty} \times U(1)^{r}
$$

Furthermore, the gauge fields satisfy an additional symmetry associated to the harmonic forms. The Hamiltonian exhibits an additional invariance, related to the symplectomorphism group $\operatorname{Sp}(2 g, \mathbb{Z})$. In the particular case of the compactified sector of target space being a 2 -torus, the symmetry becomes $\operatorname{Sp}(2, \mathbb{Z}) \simeq S L(2, \mathbb{Z})$. This is the same symmetry that arises by compactifying $I I A / S^{1}$ or, equivalently, $I I B / S^{1}$. 
As it was pointed out in [16], one way to realise this symmetry in our formalism is to observe that $\mathrm{d} X^{r}$ must satisfy, see (3),

$$
\oint_{C_{s}} \mathrm{~d} X^{r}=S_{s}^{r}
$$

where $C_{s}$ is a basis of homology defined on the 2-torus and the matrix $S_{s}^{r}$ are such that

$$
S_{r}^{t} \epsilon^{r s} S_{s}^{u}=\epsilon^{t u}
$$

when $n=1$ i.e. $S_{r}^{s} \in S L(2, \mathbb{Z})$.

In the supermembrane with fixed central charges we find area preserving diffeomorphisms which are not homotopic to the identity. These diffeomorphisms correspond to bi-holomorphisms, mapping the Teichmuller space into itself. Under this conformal mapping, the basis of harmonic one-forms transforms via an element of $S L(2, \mathbb{Z})$. As we saw in Section 2, these are area preserving diffeomorphisms for our choice of $W$.

Although $A$, cf. (5), is single-valued over $\Sigma$, it has an infinitesimal gauge transformation law that represents an unusual realisation of the diffeomorphisms algebra,

$$
A \rightarrow A+D \xi+\{A, \xi\}=A+\mathcal{D} \xi .
$$

This transformation is generated by a first class constraint at both the exact and the regularised level. See the Appendix. It corresponds to a symplectic connection, preserving the symplectic structure of the fibres under holonomies.

With this transformation, the general structure of the first class constraint which generates the gauge symmetry of the theory is that of an algebra at both the exact as well as the $S U(N)$ regularised model. We remark that the transversal modes transform in the standard way,

$$
\delta X=\{\xi, X\}
$$

In order to construct the non-commutative gauge theory, one has to fix the harmonic sector. The resulting symmetry is the centre of $\operatorname{Sp}(2, \mathbb{Z})$ which is $\mathbb{Z}(2)$.

5.2. The centre of the group as a mechanism for confinement in the exact theory. The mass contribution of the central charge, or, analogously, its correlated residual $\mathbb{Z}(2)$ symmetry of the Hamiltonian 1 , can be described in terms of the quadratic derivatives of the

\footnotetext{
${ }^{1}$ Strictly speaking the residual symmetry is a $\mathbb{Z}(2) \times \mathbb{Z}(2)$ at classical level, however at quantum level one of the $\mathbb{Z}(2)$ symmetries is not preserved by the measure
} 
configuration fields $X^{m}$ and $A_{r}$. These are induced by the minimal immersion realised by $\widehat{X}_{r}, r=1,2$, the harmonic fields over $\Sigma$,

$$
D_{r} Y_{A}=\left\{\widehat{X}_{r}, Y_{A}\right\}=\lambda_{r A}^{B} Y_{B}=\lambda_{r A} Y_{A}
$$

where

$$
\lambda_{r A}^{B}=\int d^{2} \sigma \sqrt{\omega}\left\{\widehat{X}_{r}, Y_{A}\right\} Y^{B} .
$$

They correspond to a particular subset of the structure constants that mixes the harmonic and the exact forms, $g_{r A}^{C}$. For the case of a torus, an explicit relation was found in [12]. The quadratic terms on the derivatives of the configuration variables, define a strictly positive function whose contribution to the overall Hamiltonian gives rise to a basin shaped potential. The latter eliminates the string-like spikes and provides a discrete spectrum, even for the supersymmetric model.

Without the central charge, the SUSY contribution renders a potential unbounded from below along the directions where the quartic contribution vanishes. This would produce a non-empty continuous spectrum. The quantum mass is bounded from below by the semiclassical term, see (11) and (22). Then, once the topological condition is implemented, the centre created by a discrete symmetry is a mechanism for rendering mass to the monopoles.

A natural question to ask is what happens when we compactify the target space by a larger space, say $T^{6}$. The size of the symplectic group increases. However, as explained in [38], the size of the centre of the group remains constant modulo $\mathbb{Z}(2)$, in distinction to the $S U(N)$ gauge groups. From a lattice point of view, $\operatorname{Sp}(2)$ and $\operatorname{Sp}(3)$ were used to demonstrate de-confined transition phases induced by the breaking of the centre.

5.3. Centre of the group as a mechanism for confinement in the $S U(N)$ formalism. The centre of the group in the $S U(N)$ regularisation is known to be $\mathbb{Z}_{N}$. Since the origin of this symmetry is an inherited structure of topological nature created by the monopoles induced in the torus, the real discrete symmetry is $\mathbb{Z}_{N} \times \mathbb{Z}_{N}$. The realisation of the latter in terms of the field theory should correspond to a symmetry-preserving gauge equivalent classes. Although we do not

of integration

$$
\operatorname{det}\left([\Phi, \chi]_{\text {Poisson }}\right)
$$

where $\Phi=\mathcal{D}_{r} \Pi^{r}+\left\{P_{m}, X^{m}\right\}$ and $\chi=D_{2} A_{1}$. The condition $\Phi=0$ corresponds to the first class constraint and $\chi=0$ corresponds to the gauge fixing condition. The computation of $[\Phi, X]_{\text {Poisson }}=\mathcal{D}_{1} D_{2}$ does not leave this operator invariant, leaving only the center of the group $\mathbb{Z}(2)$ as the residual symmetry. 
have the explicit form of such a map, we argue that it should exist and it should be related to the mass terms. In fact, the exact theory has a symmetry $U(1) \times U(1)$, also in the large $N$ limit

$$
\mathbb{Z}_{N} \times \mathbb{Z}_{N} \rightarrow U(1) \times U(1)
$$

and ultimately the large $N$ limit of the regularised supermembrane with central charges indeed corresponds to the exact theory. Consequently, the former should have that symmetry. An element $\widehat{z}_{r A} \in \mathbb{Z}_{N} \times \mathbb{Z}_{N}$ belongs to the centre, if it satisfies the condition

$$
\widehat{z}_{r A}^{N}=1, \quad \widehat{z}_{r A}=e^{\frac{2 \pi i\left(V_{r} \wedge A\right)}{N}} .
$$

The contributions associated to the mass are defined in terms of a regularised object found in [12]. They correspond to a specific choice of the structure constants. In terms of the $S U(N)$ basis,

$$
\widehat{\lambda}_{r A} \equiv \operatorname{Tr}\left(\left[T_{V_{r}}, T_{A}\right] T_{-V_{r}-A}\right)=2 i N \sin \left(\frac{V_{r} \wedge A}{N} \pi\right)
$$

where $T_{V_{r}}$ correspond to two particular matrices of $T_{A}$ from which the $S U(N)$ algebra can be expanded. The centre is

$$
\widehat{z}_{r A} \equiv \frac{1}{N^{4}} \operatorname{Tr}\left(T_{V_{r}} T_{A} T_{-V_{r-A}}\right) .
$$

Following [12, these matrices are given by $T_{V_{1}}=T_{0,1}$ and $T_{V_{2}}=T_{1,0}$. Here we have used that $T_{A}=N z^{1 / 2 a_{1} a_{2}} P^{a_{1}} Q^{a_{2}}$ and $T_{(0,0)}=N \mathbb{I}$ as in [4, 35]. The lambda contribution can then be re-written as

$$
\widehat{\lambda}_{r A}=\operatorname{Im}\left(\widehat{z}_{r A}\right) \text {. }
$$

The discrete mass spectrum arises in analogous way as in the exact theory.

The eigenvalues of the Hamiltonian are bounded from below by those of the semiclassical spectrum in such a way, that the mass terms are created by the centre. The unitary realisation of this centre in the Hilbert space of states, commutes with the Hamiltonian and it therefore represents an unbroken symmetry.

5.4. Confinement, screening and phase transition. As we have already seen, the mass terms are determined by the elements of the centre $m\left(\widehat{z}_{r A}\right)$ associated to $\widehat{z}_{r A}$. The matrix $T_{V_{r}}$ appears in the regularised model as a consequence of the presence of the term $\widehat{X}_{r}$. The latter are the harmonic forms associated to the winding, defining the monopole charge $\left\{\widehat{X}_{r}, \widehat{X}_{s}\right\}=\epsilon_{r s} n$. If the monopole charge disappears, the centre becomes trivial $\widehat{z_{A}}=N^{-3} \operatorname{Tr}\left(T_{A} T_{-A}\right)=N^{-2} \operatorname{Tr}\left(T_{(0,0)}\right)=1$. 
We therefore expect a breaking of the centre of the group for the deconfined phase. In a similar fashion, the same effect can be seen at the level of the exact theory.

We have then two pictures. One, corresponding to the supermembrane with central charges, in which the correlation length of the particles is the inverse of the mass of the glueball states, $\xi_{C}=1 / m_{\mathrm{eff}}$, and we can define an effective volume $V_{\text {eff }}=R / \xi_{C}$. There the particles feel the topological effects and get confined. The other one in which $m^{2}=0$ as $\xi \rightarrow \infty$ and $V_{\text {eff }}=0$. There the particles lose the information that they are confined in a boundary with topological condition, and behave as if they where in a quark-gluon plasma.

In the supersymmetric picture, the Hamiltonian corresponding to the $N=1$ supermembrane with central charges,

$$
H_{1}=\Delta_{1}+V_{1}^{B}+V_{1}^{F} \text {. }
$$

It has purely discrete spectrum at the quantum level, due to the presence of a non-trivial central charge in the algebra of supersymmetry.

In contrast, the Hamiltonian corresponding to the $N=4$ compactified supermembrane without central charges,

$$
H_{2}=\Delta_{2}+V_{2}^{B}+V_{2}^{F},
$$

has a continuum spectrum. The supermembrane is interpreted as a many-body object fluctuating into different vacua were neither the number of particles nor the topology of the membrane is preserved [2, 10]. We conjecture that this case describes the quark-gluon plasma.

Moreover, since the potential vanishes, the particles are free along the flat directions corresponding to the commutative picture. The particles do not feel any force between them, as it is expected in the asymptotic free regime of a SUSY QCD. The transition is then a consequence of the presence of a quantum change in the irreducible winding. Although both types of membranes are compact and can have the same topology, there is a change of quantum nature in the topological condition. It corresponds to the sequence

$$
U(1) \times U(1) \longrightarrow U(1) \longrightarrow \mathbb{Z}(2),
$$

related to a monopole bounding two strings.

We conjecture about the origin of this phenomenon. If we decrease the energy scale, it becomes more advantageous for the membrane to have an irreducible wrapping. Since the radius of the compactified extra dimension becomes smaller as we lower the scale, there is a critical scale at which the area of the supermembrane is minimised, not by wrapping in a cycle, but by considering a calibrated submanifold 
generated by the monopoles dual to the irreducible wrapping. For an account on the quantum topological change in $(2+1) \mathrm{D}$ see [45, 43].

\subsection{Supermembrane origin and interpretation of SUSY QCD.}

We would like to stress that, in our picture, confinement is a consequence of two issues: supersymmetry and the addition of extra dimensions. By virtue of the topological condition imposed on the extra dimensions, the supersymmetry is broken at critical energies. We believe that this topological condition appears naturally when the size of the extra dimensions decrease. It corresponds to the presence of a central charge of the supersymmetric algebra and it adds mass to the gluons entering a confined phase.

Since the magnetic flux is confined in the monopoles picture (as it was originally explained in [36]), the electric flux between them gets also confined, forming a $Z_{2}$-string at the ends of which quarks become attached [48]. In order to separate them, one needs to provide a force proportional to the force needed for increasing the effective radius of the compact dimensions. This force grows linearly with the radius. In this picture the confinement of quarks is then consequence of the compactification of extra dimensions and the fact that supermembrane has an irreducible wrapping around them.

Separating the gluons means de-compactifying the space. In higher energies, the size of the effective radius of the extra dimensions becomes bigger. At those energies the supermembrane does not minimise its energy with an irreducible wrapping around it (which corresponds to wrap a calibrated submanifold), but just wraps in cycles that minimise their volume.

It is known that the presence of topological defects can diminish the energy of the vacuum. This is what happens in our case. Without these defects, the compactification process allows degenerate points on the metric. Changes in the metric and the topology are also allowed in the classical analysis of GR [46]. This issue has been studied in [45, 43, 44.

A change in the topology [45] leads then to a loss of the monopole picture. The centre of the group becomes trivial, hence the theory enters in a quark-gluon plasma phase. In the latter state, the supermembrane can not be associated to a single particle but rather to a multiple body object in a potential of continuous spectrum. The theory contains free quarks along the commutative directions that corresponds to a vanishing potential.

Since the correlation length becomes infinite and the effective volume is zero, the quarks-gluons form a plasma that does not feel the boundary 
effects. This corresponds to seing inside the hadron, i.e. at a shorter scale.

A further natural explanation emerges from here. Supersymmetry is where the topological condition originates. We conjecture that, perhaps, this is the way in which supersymmetry breaking realises. This type of compactification does not produce exotic matter as in the KK reduction, but rather gives mass (without a Higss mechanism) to the scalar fields as the supersymmetry is broken. We may speculate that supersymmetry, membrane description and the addition of extra dimensions, would be the reason for a QCD behaviour in both phases: the confined one and the quark-gluon plasma one.

\section{Discussion AND CONCLUSIONS}

Our results provide bounds, in the operator sense, for the bosonic Hamiltonian of the $D=11$ supermembrane with central charges. We have shown that this Hamiltonian is bounded from below by a strictly positive constant, times the Hamiltonian of coupled harmonic oscillators. We have then shown that the spectrum of the Hamiltonian is discrete with finite multiplicity and it contains a mass gap. This result extends to the exact theory the bound already obtained for the $S U(N)$ regularised model. We are not aware of any other similar analysis in the literature, regarding the spectrum of the exact supermembrane theory rather than that of the $S U(N)$ regularised theory. Our bound gives strong evidence that the quantum properties of the supersymmetric potential will not change qualitatively those of the bosonic potential.

In previous works we have analysed the heat kernel of the regularised supermembrane with central charges. Convergence of the kernel's asymptotic expansion was obtained in the Schatten-von Neumann norms, implying a well defined Feynman formula. The large $N$ limit of such formula is expected to converge to the Feynman integral of the supermembrane with central charges.

Since this theory is the quantum equivalent of a symplectic NCSYM theory in $2+1$ dimensions, the same spectral properties are valid for the latter. The symplectic NCSYM in $2+1$ dimensions is coupled to scalar fields arising from the dimensional reduction of NCYM in $9+1$ dimensions. The number of degrees of freedom of both theories coincides. In this context, our results provide a one-step-forward towards the quantisation of M-theory.

We have also argued that the supermembrane theory, when compactified in 4-D, can be interpreted as a theory modelling SUSY QCD. As the theory becomes the $N=1$ supermembrane with central charges at 
zero temperatures, it exhibits confinement in the phase. By rising the energy, the theory enter in a phase of asymptotic freedom described by the $N=4$ compactified supermembrane without central charges. We have shown that the phase transition is described by the breaking of the centre of the group.

We have also conjectured a possible reason for this phase transition. At high energies, the size of the effective radius of the extra dimensions becomes bigger. In the same regime, the irreducible wrapping of the supermembrane on a calibrated submanifold of the target, turns into a reducible wrapping in the compact sector of the target space with zero central charge. This corresponds to seeing inside the hadron, that is at a shorter scale. Since the correlation length becomes infinite and the effective volume is zero, the quarks-gluons form a plasma that does not feel the topological effects. Along the commutative directions, the quarks experience no force.

\section{Appendix}

Here we compute explicitly the $S U(N)$ gauge symmetry in the regularisation of the $D=11$ supermembrane with central charges.

The general structure of the first class constraints which generate the gauge symmetry of the regularised model arising from the $D=11$ supermembrane with central charges is,

$$
\phi^{D} \equiv \lambda f_{E-w_{s}, w_{s}}^{D} \Pi^{E s}+f_{A+w_{s}, F-w_{s}}^{D} A_{s}^{A} \Pi^{F s}=0 .
$$

The constant $\lambda$ is an arbitrary parameter and $f_{A B}^{C}$ are the $S U(N)$ structure constants.

The algebra associated to the first class constraints is obtained by considering the Poisson brackets of the generators. We have,

$$
\begin{aligned}
{\left[\lambda f_{E-w_{s}}^{D},\right.} & \left.w_{s} \Pi^{E s}+f_{A+w_{s}, F-w_{s}}^{D} A_{s}^{A} \Pi^{E s}, f_{L-w_{r}}^{C}, w_{r} \Pi^{L r}+f_{F+w_{r}, E-w_{r}}^{C} A_{r}^{F} \Pi^{E r}\right]_{P}= \\
& \lambda f_{E-w_{s}, w_{s}}^{D} f_{-E+w_{s}, F-w_{s}}^{C} \Pi^{F s}-\lambda f_{-L+w_{s}, F-w_{s}}^{D} f_{L-w_{r}, w_{r}}^{C} \Pi^{F s} \\
& +f_{A+w_{s}, E-w_{s}}^{D} f_{-E+w_{s}, F-w_{s}}^{C} A_{s}^{A} \Pi^{F s}-f_{E+w_{s}, F-w_{s}}^{D} f_{A+w_{s},-E-w_{s}}^{C} A_{s}^{A} \Pi^{F s} .
\end{aligned}
$$

We now use the explicit expression for the structure constants. For the terms depending on $\lambda$ we obtain

$$
\lambda \delta_{F}^{D+C} \Pi^{F s} N^{2} \sin \left(\frac{(C \wedge D)}{N} \pi\right) \sin \left(\frac{(D+C) \wedge w_{s}}{N} \pi\right)
$$


which may be re-written as $\lambda f_{-D, E}^{C} f_{F-w_{s}, w_{s}}^{E} \Pi^{F s}$. For the remaining terms we obtain the expression

$$
\begin{aligned}
N^{2} \delta_{A+F}^{C+D} & \sin \left(\frac{\left(A+w_{s}\right) \wedge D}{N} \pi\right) \sin \left(\frac{C \wedge\left(F-w_{s}\right)}{N} \pi\right) \\
& -\sin \left(\frac{D \wedge\left(F-w_{s}\right)}{N} \pi\right) \sin \left(\frac{\left.\left(A+w_{s}\right) \wedge C\right)}{N} \pi\right),
\end{aligned}
$$

which may be re-written as $f_{-D, E}^{C} f_{A+w_{s}, F-w_{s}}^{E} A_{s}^{A} \Pi^{F s}$. Thus, for any $\lambda$ and $N$,

$$
\left[\phi^{D}, \phi^{C}\right]_{\text {Poisson }}=f_{-D, E}^{C} \phi^{E}
$$

where $f$ are the $S U(N)$ structure constants.

The analogous computation is valid as we take the large $N$ limit. In fact, by considering the same constraints, but with the structure constants associated to the area preserving diffeomorphisms, we obtain the corresponding algebra (29).

As $N \rightarrow \infty$, we find an equivalent realisation of the generators in terms of the constraints,

$$
\widetilde{\phi}^{D}=\lambda f_{E-w_{s}, w_{s}}^{D} \Pi^{E s}+f_{A, F}^{D} A_{s}^{A} \Pi^{F s}=0 .
$$

They also satisfy

$$
\left[\widetilde{\phi}^{D}, \widetilde{\phi}^{C}\right]_{\text {Poisson }}=f_{-D, E}^{C} \widetilde{\phi}^{E}
$$

As a particular case, we can take $\lambda=1$.

In the regularised model analysed in [12], we wrote the exact model in terms of the decomposition on an orthonormal basis over the Riemann surface. For this, we fixed the gauge and then obtained a regularised model. An alternative approach is to obtain the regularised model satisfying the symmetry generated by $\phi^{D}$ and then perform the gauge fixing.

\section{ACKNOWLEDGEMENTS}

We are very grateful to M. Asorey, F. Cachazo, I. Martin, H. Nicolai and Sh. Matsuura for helpful discussions and comments. We are also indebted to C. Burgess, F. Cachazo, R. Myers and F. Quevedo, for their support and encouragement. M.P.G.M. and A.R. would like to thank the Perimeter Institute for its kind hospitality where part of this work was carried out. M.P.G.M acknowledge support from NSERC Canada, MEDT Ontario and the NSERC Discovery grants program. The work of A.R. was supported by PROSUL, under contract CNPq 490134/2006-08. 


\section{REFERENCES}

[1] E. Bergshoeff, E. Sezgin, P.K. Townsend, Supermembranes and elevendimensional supergravity. Phys. Lett. B189: 75-78, 1987.

[2] B. de Wit, M. Luscher, H. Nicolai, The supermembrane is unstable. Nucl. Phys. B320: 135, 1989.

[3] B. de Wit, U. Marquard, H. Nicolai, Area preserving diffeomorphisms and supermembrane lorentz invariance. Commun. Math. Phys. 128: 39-62, 1990.

[4] B. de Wit, J. Hoppe, H. Nicolai, On the quantum mechanics of supermembranes. Nucl. Phys. B305: 545,1988.

[5] J. Hoppe, Ph.D Thesis, MIT, 1982.

[6] M. Claudson, M. B. Halpern, Supersymmetric ground state wave functions. Nucl. Phys.B250: 689, 1985.

[7] M. Lüscher, Some analytic results concerning the mass spectrum of Yang-Mills gauge theories on a torus. Nucl.Phys. B219: 233-261, 1983.

[8] M.P. Garcia del Moral, L. Navarro, A.J. Perez, A. Restuccia, Intrinsic moment of inertia of membranes as bounds for the mass gap of Yang-Mills theories. Nucl. Phys.B765: 287-298, 2007. hep-th/0607234

[9] B. de Wit, K. Peeters, J. Plefka, Supermembranes with winding. Phys. Lett. B409: 117-123, 1997. hep-th/9705225

[10] H Nicolai, R Helling, Supermembranes and matrix theory. 1998. hep-th/9809103

[11] I. Martin, A. Restuccia, R. S. Torrealba, On the stability of compactified $D=$ 11 supermembranes. Nucl. Phys. B521: 117-128, 1998. hep-th/9706090

[12] M.P. Garcia del Moral, A. Restuccia, On the spectrum of a noncommutative formulation of the $D=11$ supermembrane with winding Phys.Rev. D66 045023, 2002. hep-th/0103261

[13] L. Boulton, M. P. Garcia del Moral, I. Martin, A. Restuccia On the spectrum of a matrix model for the $D=11$ supermembrane compactified on a torus with non-trivial winding. Class. Quant. Grav. 19 2951, 2002. hep-th/0109153

[14] L. Boulton, M.P. Garcia del Moral, A. Restuccia, Discreteness of the spectrum of the compactified $D=11$ supermembrane with non-trivial winding. Nucl.Phys. B671 343-358, 2003. hep-th/0211047

[15] L. Boulton and A. Restuccia, The Heat kernel of the compactified $D=11$ supermembrane with non-trivial winding. Nucl. Phys. B724 380-396, 2005. hep-th/0405216

[16] J. Bellorin, A. Restuccia, D=11 Supermembrane wrapped on calibrated submanifolds Nucl.Phys. B737 190-208, 2006. hep-th/0510259

[17] M.P. Garcia del Moral, A. Restuccia, The Supermembrane with central charge as a bundle of D2 - D0 branes. Institute of Physics Conference Series 2005, Vol 43, 151. hep-th/0410288

[18] R. Gianvittorio, A. Restuccia, J. Stephany, Interacting D2-branes in 10 dimensions and non Abelian Born-Infeld theory. Class. Quant. Grav. 23: 7471-7478, 2006. hep-th/0606063

[19] I. Martin, J. Ovalle, A. Restuccia, D-branes, symplectomorphisms and noncommutative gauge theories. Nucl. Phys. Proc. Suppl. 102: 169-175, 2001; Compactified $D=11$ supermembranes and symplectic noncommutative gauge theories. Phys. Rev.D64: 046001, 2001. hep-th/0101236 
[20] I. Martin and A. Restuccia, Symplectic connections, noncommutative Yang-Mills theory and supermembranes. Nucl.Phys. B622: 240-256, 2002. hep-th/0108046

[21] D. Gross, A. Hashimoto, N. Itzhaki, Observables of noncommutative gauge theories. Adv. Theor. Math. Phys. 4: 893-928, 2000. hep-th/0008075

[22] R.J. Szabo, Symmetry, gravity and noncommutativity. Class. Quant. Grav.23: R199-R242, 2006. hep-th/0606233

[23] L. Cornalba, R. Schiappa, Nonassociative star product deformations for Dbrane world volumes in curved backgrounds. Commun. Math. Phys.225: 33-66, 2002. hep-th/0101219

[24] A. Connes, M. Douglas, A. Schwarz, Noncommutative geometry and matrix theory: Compactification on tori. JHEP 9802: 003, 1998. hep-th/9711162

[25] Miao Li, Comments on supersymmetric Yang-Mills theory on a noncommutative torus. 1998. hep-th/9802052

[26] M. Douglas, C. Hull, D-branes and the noncommutative torus. JHEP 9802: 008,1998. hep-th/9711165

[27] N. Ishibashi, A Relation between commutative and noncommutative descriptions of D-branes. Beijing Frontiers of theoretical physics, 99-109, 1999, and Hayama Noncommutative differential geometry and its applications to physics 49-61, 1999. hep-th/9909176

[28] D. Bigatti, L. Susskind, Noncommutative geometry and super Yang-Mills theory. Phys. Lett. B451: 324-335, 1999. hep-th/9804120

[29] J. M. Maldacena, J. G. Russo, Large N limit of noncommutative gauge theories. JHEP 9909: 025, 1999. hep-th/9908134

[30] A. Hashimoto, N. Itzhaki, On the hierarchy between noncommutative and ordinary supersymmetric Yang-Mills. JHEP 9912: 007, 1999. hep-th/9911057

[31] T. Banks, W. Fischler, S.H. Shenker, L. Susskind, M Theory as a matrix model: a conjecture. Phys. Rev. D55 5112-5128, 1997. hep-th/9610043

[32] D. Berenstein, J. M. Maldacena, H. Nastase, Strings in flat space and pp waves from N=4 superYang-Mills. JHEP 0204: 013, 2002. hep-th/0202021

[33] M. Pepe, Confinement and the center of the gauge group. Nucl. Phys. Proc. Suppl. 153: 207-214, 2006. hep-lat/0510013

[34] Kuo Hui Hsiung Integration in Banach space. Notes in Banach spaces, pp. 1-38, Univ. Texas Press, Austin, Tex., 1980.

[35] G. t'Hooft, On the phase transition towards permanent quark confinement. Nucl. Phys. B138: 1, 1978.

[36] G. 't Hooft, Topology of the gauge condition and new confinement phases in nonabelian gauge theories. Nucl. Phys. B190: 455, 1981.

[37] O. Aharony, E. Witten, Anti-de Sitter space and the center of the gauge group. JHEP 9811: 018, 1998. hep-th/9807205

[38] K. Holland, M. Pepe, U.J. Wiese, The Deconfinement phase transition of Sp(2) and Sp(3) Yang-Mills theories in (2+1)-dimensions and $(3+1)$-dimensions. Nucl. Phys. B694: 35-58, 2004. hep-lat/0312022

[39] E. Witten, Instantons, the quark model and the $1 / N$ expansion. Nucl. Phys. B149: 285-320, 1979.

[40] G. Gabadadze, Modeling the glueball spectrum by a closed bosonic membrane. Phys. Rev. D58: 094015, 1998. hep-ph/9710402 
[41] A. Chodos, R.L. Jaffe, K. Johnson, Charles B. Thorn, V.F. Weisskopf, A new extended model of hadrons. Phys. Rev. D9: 3471-3495, 1974.

[42] B. Svetitsky, L. G. Yaffe, Critical Behavior At Finite Temperature Confinement Transitions. Nucl.Phys.B210: 423, 1982.

[43] J.Preskill, A. Vilenkin, Decay of metastable topological defects. Phys. Rev. D47: 2324-2342, 1993. hep-th/9209210

[44] A. S. Kronfeld, G. Schierholz, U.J. Wiese, Topology And Dynamics Of The Confinement Mechanism. Nucl. Phys. B293: 461, 1987.

[45] A.P. Balachandran, E. Batista, I.P. Costa e Silva, P. Teotonio-Sobrinho, Quantum topology change in (2+1)-dimensions. Int. J. Mod. Phys. A15: 1629-1660, 2000. hep-th/9905136

[46] G. T. Horowitz, Topology change in classical and quantum gravity. Class. Quant. Grav. 8: 587-602, 1991.

[47] M.J. Duff, T. Inami, C.N. Pope, E. Sezgin, K.S. Stelle, Semiclassical Quantization Of The Supermembrane. Nucl. Phys. B297: 515, 1988.

[48] Marco A.C. Kneipp, Z(k) string fluxes and monopole confinement in nonAbelian theories. Phys. Rev. D68: 045009, 2003. hep-th/0211049 
${ }^{1}$ Department of Mathematics and the Maxwell Institute for Mathematical Sciences, Heriot-Watt University, Edinburgh EH14 2AS, United Kingdom. email: L.Boulton@hw.ac.uk

${ }^{2}$ Perimeter Institute for Theoretical Physics, Waterloo, Canada, Ontario N2L 2Y5, Canada.

Dept. of Physics and Astronomy, MacMaster University, 1280 Main Street West, Hamilton, Ontario, L8S 4M1, Canada.

DAMTP, DAMTP, Centre for Mathematical Sciences, University of Cambridge, Cambridge CB3 OWA, United kingdom.

emails: mmoral@perimeterinstitute.ca

M.G.d.Moral@damtp.cam.ac.uk

${ }^{3}$ Departamento de Física, Universidad Simón Bolívar, Apartado 89000, Caracas 1080-A, Venezuela.

email: arestu@usb.ve 